\title{
CONTAMINATION OF GROUND-BASED MEASUREMENTS OF OI ( $6300 \AA)$ AND NI (5200 Å) AIRGLOW BY OH EMISSIONS
}

\author{
(Received in final form 8 March 1977)
}

\begin{abstract}
High resolution spectra of the $6300 \AA$ and $5200 \AA$ regions of the night sky have been obtained using a $1 \mathrm{~m}$ spectrometer. Typical errors in measurements of $O\left({ }^{1} \mathrm{D}\right) 6300 \AA$ and $N\left({ }^{2} \mathrm{D}\right)$ $5200 \AA$ intensities due to contanimation by overlapping $\mathrm{OH}$ emissions have been calculated for a fixed-filter photometer, a tilting-filter photometer and a spectrophotometer. The importance of careful selection of certain instrumental parameters in order to minimize measurement errors is emphasized.
\end{abstract}

\section{INTRODUCTION}

The forbidden transitions of atomic oxygen and atomic nitrogen, $6300 \AA\left(\mathrm{O}^{1} \mathrm{D} \rightarrow \mathrm{O}^{3} \mathrm{P}\right)$ and $5200 \AA\left(\mathrm{N}^{2} \mathrm{D} \rightarrow \mathrm{N}^{4} \mathrm{~S}\right)$, are weak airglow emissions that can provide valuable insight into the nighttime behaviour of the $F$ region. However, the $\mathrm{OH}$ airglow emission in the mesosphere poses serious contamination problems for the accurate measurement of any airglow emission by optical groundbased instruments limited to a spectral resolution of a few Angstroms. The airglow atlas of Broadfoot and Kendall (1968) illustrates the great proliferation of the $\mathrm{OH}$ molecular bands throughout the night sky spectrum. The high resolution spectra obtained by Hernandez and Turtle (1969) and Hernandez (1974) document for $5200 \AA$ and $6300 \AA$, respectively, the nature of this contamination. However, it is difficult to judge from these spectra the extent of the $\mathrm{OH}$ contamination.

For a Fabry-Perot interferometer, there may be as many as $\mathbf{2 5}$ orders within the transmission passband of the interference filter used as a monochromator to isolate the spectral feature of interest. Each order contains the contaminating $\mathrm{OH}$ rotational line and the $F$ region airglow feature, but the intensity of each varies according to the order, and depends upon the filter transmission of the airglow line at the wavelength of the order being scanned. The wavelength of the filter passband is chosen to optimize the transmission of the $F$ region airglow feature and to minimize the $\mathrm{OH}$ airglow contamination by attenuation. Therefore, the true intensity of the $\mathrm{OH}$ component is often not obvious from Fabry-Perot spectra. Furthermore, unless one also knows the number of orders separating the $\mathrm{OH}$ component from the $F$ region feature, it is not possible to determine from a Fabry-Perot spectrum the wavelength of the $\mathrm{OH}$ line, but only its relative separation from the $F$ region airglow line within one order. Therefore, the high resolution Fabry-Perot spectrum does not provide a useful display of the $\mathrm{OH}$ contamination in either wavelength or intensity.

The seriousness of the $\mathrm{OH}$ contamination problem and the difficulty it presents in the accurate determination of the 5200 and $6300 \AA$ airglow intensities have been known for many years. While the problem is not so serious as to preclude the use of the instruments most commonly employed, which are fixed-filter photometers, tilting-filter photometers and spectrophotometers, this problem does require a careful study (with a monochromator) of the instrumental transmission as a tunction of wavelength. Although this may seem obvious, we feel that little attention in recent years has been given to this aspect of airglow observations. Papers have been pub- lished concerning airglow observations that give no discussion of a particular instrument's ability to reject the $\mathrm{OH}$ contamination. To remind everyone that this $\mathrm{OH}$ contamination problem still remains, we show spectra of the 5200 and $6300 \AA$ spectral regions with a resolution of approximately $1.5 \AA$. These permit unambiguous assessment of the OH contamination with respect to wavelength and intensity.

We use these values of $\mathrm{OH}$ intensity to determine the error of intensity measurements for both $\$ 200$ and $6300 \AA$ as a function of instrumental bandwidth and the intensity of the $F$ region airglow emission. From this study we conclude that for the $5200 \AA$ spectral feature the $\mathrm{OH}$ contamination is very serious and may never be ignored. We find that measurements of $6300 \AA$ intensities can be attained with errors less than $10 \%$ if the instrumental bandwidth is less than $5 \AA$ and if the $6300 \AA$ intensity is greater than $20 \mathrm{R}$.

\section{OH CONTAMINATION OF $6300 \AA$ MEASUREMENTS}

Figure 1 is a zenith spectrum of the $6300 \AA$ region obtained after integration for $90 \mathrm{~min}$ at Arecibo at a time when the $6300 \AA$ intensity was fairly low (33 R). The instrument used was a $1 \mathrm{~m}$ Ebert-Fastie spectrophotometer at a spectral resolution of $1.5 \AA$. This figure shows the $P_{1}$ (3), $P_{2}$ (3) and the $P_{1}(2)$ rotational components of the OH 9-3 band to be present with intensities of $4.9,2.6$ and $4.2 \mathrm{R}$, respectively. In Table 1 these results are seen to be comparable with those reported by Hernandez (1974).

The spectral features shown in Fig. 1 were convolved with a number of different instrumental transmission profiles. For the fixed-filter and the tilting-filter photometer simulations, the transmission curve of an interference filter was assumed to be Gaussian, i.e.

$$
T_{\lambda}=T_{0} \exp \frac{-2.77\left(\lambda-\lambda_{0}\right)^{2}}{B^{2}},
$$

where $\lambda_{0}$ is the peak wavelength, $T_{0}$ the peak transmission and $B$ the bandwidth at $50 \%$ transmission.

From the synthetic spectra resulting from the convolution the fractional error in any $6300 \AA$ measurement was calculated using,

$$
\epsilon=\left(R-R^{\prime}\right) / R^{\prime},
$$

where $R$ and $\boldsymbol{R}^{\prime}$ are the observed peak signals when the $\mathrm{OH}$ intensities are non-zero and equal to zero respectively. 


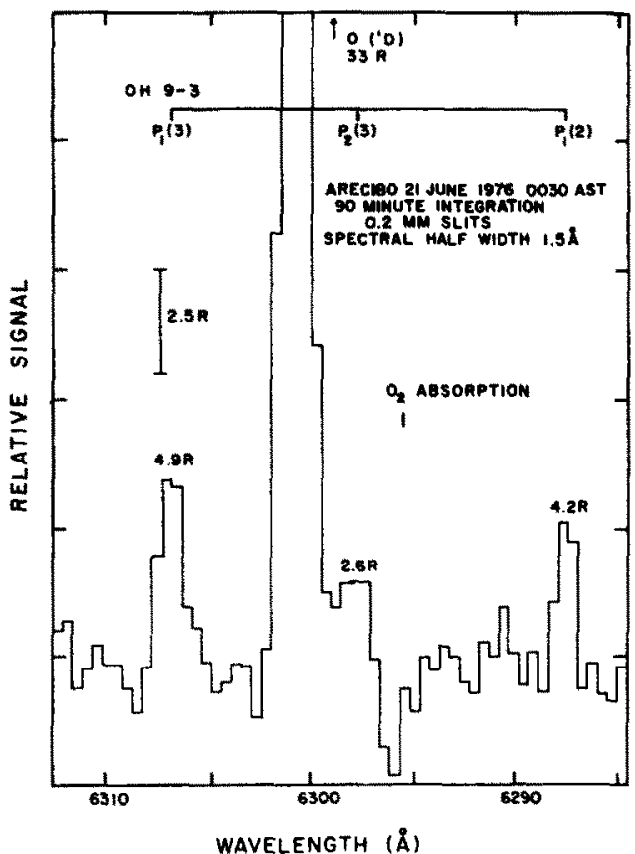

Fig. 1. HIGH Resolution ZENTTH SPECTRUM OF THE $6300 \AA$ REGION OBTAINED WITH THE ONE METRE EDERTFASTIE SPECTROPHOTOMETER BY THE SUMMATION OF 570 SCANS.

The error curves shown in Fig. 2(a) apply to a fixedfilter photometer with a filter centred at $6300.3 \AA$. The same set of curves also apply to the case of a tilting-filter photometer using a filter positioned with zero tilt angle at $6300.3 \AA$. However, in the tilting-filter system, when the filter is tilted the photometer will scan through the $P_{2}(3)$ and $P_{1}(2) O H$ lines, leading to a possibility of error in the determination of the background level. This error should be minimized by measuring the background at a wavelength well removed from that of any $\mathrm{OH}$ emissions. For the same reason the background measurement should not be made in the region of the $0-2 \mathrm{O}_{2}\left(b^{\prime} \Sigma_{g}+-X^{3} \Sigma_{g}-\right)$ absorption band located near $6295 \AA$ (Curcio et al., 1964). The same method was used to examine the $O H$ contamination errors when a spectrophotometer replaces

Tarle 1. Emissions IN THE $6300 \AA$ Region

\begin{tabular}{lccc}
\hline \multicolumn{1}{c}{ Transition } & $\begin{array}{c}\text { Wave- } \\
\text { length }(\AA)^{*}\end{array}$ & $\begin{array}{c}\text { Typical } \\
\text { intensity } \\
(\mathbf{R})\end{array}$ & $\begin{array}{c}\text { Measured } \\
\text { intensity } \\
(\mathbf{R}) \neq\end{array}$ \\
\hline OH $(9-3), \mathrm{P}_{1}(2)$ & 6287.4 & $5.75 \dagger$ & 4.2 \\
OH $(9-3), \mathrm{P}_{2}(3)$ & 6298.0 & $2.0-4.0^{*}$ & 2.6 \\
OI $\left({ }^{3} \mathrm{P}_{2}-{ }^{1} \mathrm{D}_{2}\right)$ & 6300.3 & variable & variable \\
OH $(9-3), \mathrm{P}_{1}(3)$ & 6306.8 & $5.0-8.0^{*}$ & 4.9 \\
\hline
\end{tabular}

*From Hernandez (1974).

†Estimated from typical relative intensities.

$\ddagger$ Arecibo $1 \mathrm{~m}$ spectrometer.

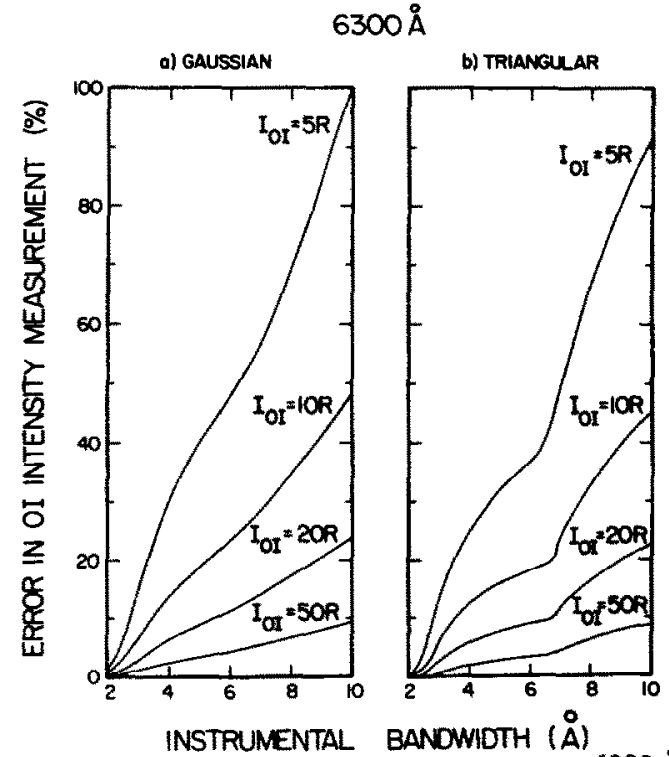

Fig. 2. The OH contramination errors of $6300 \AA \mathrm{IN}$. TENSITY MEASUREMENTS AS A FUNCTION OF INSTRUMENTAL BANDWIDTH ASSUMING (a) A GAUSSIAN TRANSMISSION PROFILE (FILTER PHOTOMETER) AND (b) A TRIANGULAR PROFILE (SPECTROPHOTOMETER).

The error curves corresponding to 4 different values of $O\left({ }^{1} D\right)$ intensity are shown.

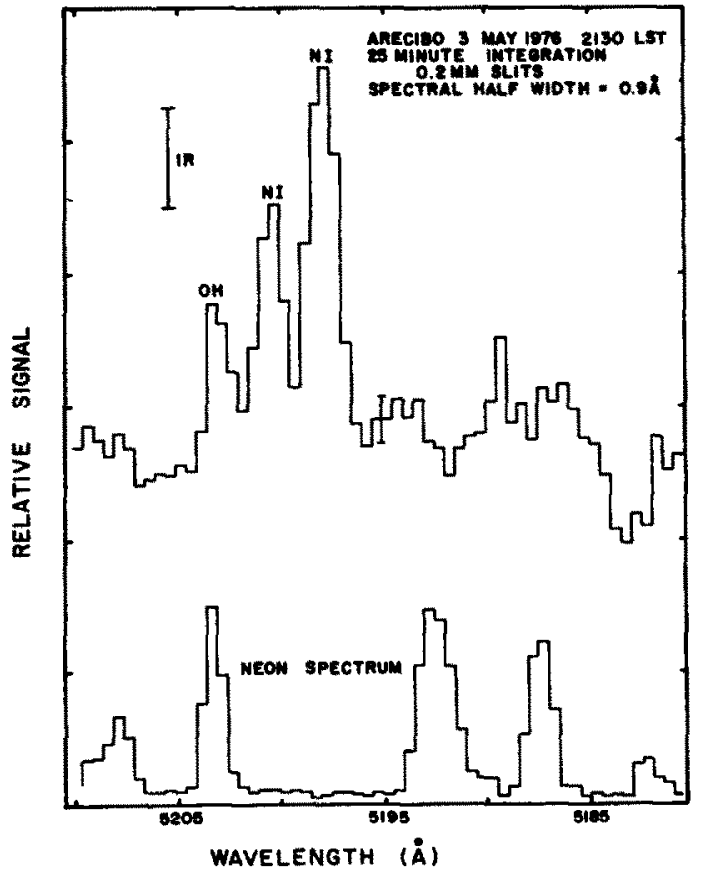

FIG. 3. HIGH RESOLUTION SPECTRUM OF THE $5200 \AA$ REGION PRODUCED BY THE SUMMATION OF 180 SCANS.

The one metre spectrometer was oriented westwards at $60^{\circ}$ zenith angle. A neon wavelength calibration spectrum is also shown. 
the interference filter. For this case the instrumental transmission was represented by a triangular function given by,

$$
T_{\lambda}=T_{o}-\left\{\frac{T_{\mathrm{o}}\left|\lambda-\lambda_{\mathrm{o}}\right|}{B}\right\},\left|\lambda-\lambda_{\mathrm{o}}\right| \leq B
$$

and

$$
T_{\lambda}=0,\left|\lambda-\lambda_{0}\right|>B .
$$

The magnitude of the $\mathrm{OH}$ contamination error is slightly reduced when a triangular transmission function is assumed. The error curves shown in Fig. 2(b) apply to scanning spectrometer measurements.

\section{OH CONTAMINATTON OF $5200 \AA$ AEASUREMENTS}

Figure 3 is a spectrum of the $5200 \AA$ region at a spectral resolution of $0.9 \AA$ obtained at Arecibo with a one metre Ebert-Fastie spectrophotometer during late evening twilight. The integration time required was $25 \mathrm{~min}$ and the elevation angle was $30^{\circ}$. The intensities of the $\mathrm{OH} \mathrm{Q}_{1}(1)$ line at 570) $2.8 \AA$ and the $N\left({ }^{2} \mathrm{D}\right)$ doublet components at 5200.4 $\AA$ and $5198.1 \AA$ are $1.7,2.7$ and 4.1 R, respectively. The branching ratio of the two components of the $N\left({ }^{2} \mathrm{D}\right)$ doublet is $1.52 \pm 0.1$ which agrees with the theoretical value of 1.56 (Garstang, 1956) and the experimental value of $1.56 \pm \dot{0} .1$ (Hernandez and Turtle, 1969). Some components of the $R$ branch of the $\mathrm{OH}$ 9-2 band near $5190 \AA$ can also be seen. In the course of a night's observations the ratio between the $N\left({ }^{2} \mathrm{D}\right)$ doublet intensity and the $Q_{1}(1) \mathrm{OH}$ line at Arecibo has been observed to change, being between 5 and 10 at evening twilight, between 2 and 5 during any nighttime enhancements such as the post-midnight collapse, and of the order of 1 or lower at other times. Therefore, our simulation analysis of the error introduced by $\mathrm{OH}$ contamination includes several values for this ratio to encompass the range of error that might be present in $5200 \AA$ airglow observations on any one night.

\section{(a) The fixed-filter system}

As a typical cxample, the crrors which may result from the use of a filter centred at $5200.0 \AA$ are shown in Fig. 4(a). However, this would not be a good choice because a larger signal due to the $N\left({ }^{2} \mathrm{D}\right)$ doublet would be measured by a filter centred at a wavelength of $5199.0 \AA$. However, by centering the filter on the stronger $N\left({ }^{2} \mathrm{D}\right)$ line at $5198.1 \AA$, the magnitude of the $\mathrm{OH}$ contamination could be reduced even further. When this is done the errors are as shown by the dashed curves in Fig. 4(a). It should be noted that if narrow filters are used, account must be taken of the fact that the filter will probably not transmit both lines of the $N\left({ }^{2} D\right)$ doublet at the same transmission. This effect must be included in the data reduction formulae.

\section{(b) The tilting-filter system}

One method of reducing tilting-filter data is to measure the signal at the peak which is sometimes observed when the filter scans through the $5200 \AA$ region. In order to carry out an error analysis we have assumed a filter positioned at $5200.4 \AA$ for zero tilt angle with a peak transmission that drops linearly with the wavelength shift to $70 \%$ of its maximum value at $5198.1 \AA$. We have also assumed that the product of peak transmission and bandwidth remains constant, so that the bandwidth increases by $43 \%$ over this region. Filter passbands for different

$5200 \AA$

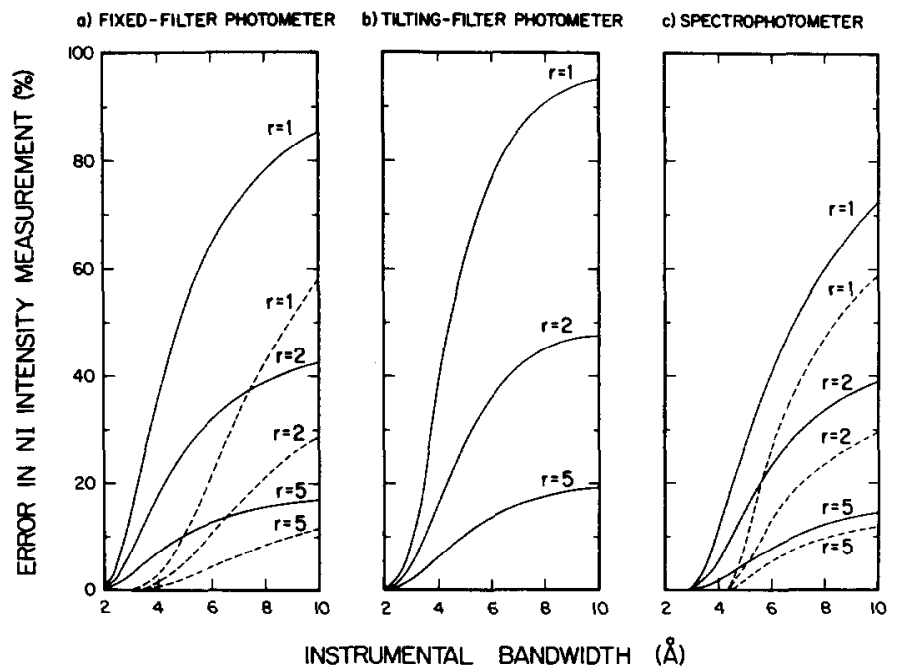

Fig. 4. THE $5200 \AA$ INTENSITY CONTAMINATION ERROR AS A FUNCTION OF INSTRUMENTAL BANDWIDTH FOR THREE INSTRUMENTS: (a) A FIXED-FLLTER PHOTOMETER USINO A FILTER CENTRED AT $5200.0 \AA$ (FULL CURVES) AND $5198.1 \AA$ (DASHED CURVES), (b) A TILTING-FILTER PHOTOMETER AND (c) A SPECTROPHOTOMETER EMPLOYING THE METHOD OF READING THE PEAK SIGNAL (FULL CURVES) AND READING THE SIGNAI AT A FIXED WA VEI ENGTH (DASHED CURVES).

The quanity " $r$ " is the ratio of the $N\left({ }^{2} D\right)$ doublet intensity to the $O H Q_{1}(1)$ intensity. 
values of the wavelength shift were convolved with the $N\left({ }^{2} D\right)$ emission spectrum in order to determine the wavelength at which the maximum signal would be observed. This wavelength is found to be a function of bandwidth which converges to $5200.4 \AA$ as the flatposition bandwidth approaches $10 \AA$.

Because the maximum signal occurs at a filter wavelength closer to the $\mathrm{OH}$ line than in the case of the $5198.1 \AA$ fixed-filter method, OH contamination produces a larger error in this tilting-filter system, as shown in Fig. 4(b). It should be appreciated that this particular casc has becn chosen as a typical cxample only. The magnitude of the $\mathrm{OH}$ contamination error is closely dependent on the variation in filter parameters with tilt angle, necessitating separate calculations of the error for every unique case. We note that even more serious errors could occur if the filter scan starts above $5203 \AA$. When the $\mathrm{OH} / \mathrm{N}\left({ }^{2} \mathrm{D}\right)$ ratio is high, the wavelength of maximum signal may be closer to the $\mathrm{OH}$ line than the $N\left({ }^{2} \mathrm{D}\right)$ doublet. When the filter is tilted in order to determine the background signal the $R$ components of the $\mathrm{OH}$ 9-2 band near $5190 \AA$ may be observed. A background measurement near this wavelength should be avoided.

\section{(c) The scanning spectrometer system}

For a spectrometer the instrumental function is triangular and the spectral halfwidth and transmission function are independent of wavelength over the narrow region of interest. In Fig. 4(c) we show the $\mathrm{OH}$ contamination errors resulting from two methods of scaling the spectrum. The full curves result if the peak reading is always used, irrespective of the wavelength at which it occurs. These results are not very useful when both the spectral width and $\mathrm{OH} / \mathrm{N}\left({ }^{2} \mathrm{D}\right)$ ratio are high, because the peak wavelength is observed near the $\mathrm{OH}$ wavelength of $5202.8 \AA$. The results of the other method, the case in which the reading was always made at $5198.1 \AA$ regardless of the position of the peak signal, are shown by the dashed set of curves in Fig. 4(c).

\section{DISCUSSION}

We conclude from Fig. 2 that filter photometry of the $6300 \AA$ nightglow to an accuracy of $10 \%$ is generally possible if the filter bandwidth is less than $5 \AA$. However, considerable care must be taken in interpreting $6300 \AA$ intensities which are observed to be less than $20 \mathrm{R}$. The simultaneous use of a filter centred on a strong $\mathrm{OH}$ line in the $9-3$ band would enable the variability of the $\mathrm{OH}$ contamination to be assessed and a correction to be made.

It follows from Fig. 4 that the fixed-filter method could be used for $5200 \AA$ work, if the filter bandwidth were $4 \AA$ or narrower. However, elaborate precautions would have to be taken to prevent a drift in the filter centre wavelength.

We recommend the calibration of the transmission profile of the filter by a monochromator on each night of observation to ensure accurate knowledge of the peak wavelength and bandwidth of the filter. Nevertheless, the errors introduced by using a different filter to measure the background level make this method a poor choice for accurate quantitative work on the $\mathrm{N}\left({ }^{2} \mathrm{D}\right)$ doublet.
The tilting-filter method allows a more accurate background subtraction, but for measurements of the $5200 \AA$ emission, the blending of the $\mathrm{N}\left({ }^{2} \mathrm{D}\right)$ doublet components with the $\mathrm{OH} \mathrm{Q}_{1}(1)$ rotational line, coupled with the change in filter characteristics with tilt angle, makes the data reduction a complicated task. Apart from during the post-twilight decay and periods of marked intensity enhancement of the $\mathrm{N}\left({ }^{2} \mathrm{D}\right)$ emission, the potential errors in tilting-filter photometry of the $5200 \AA$ doublet would suggest that this method is unsuitable for accurate work.

By using a scanning spectrophotometer of the EbertFastic type with a resolution better than $3 \AA$ the error due to $\mathrm{OH}$ contamination becomes negligible. As the temporal resolution of the Arecibo one metre spectrometer is almost comparable with that of most filter photometers we believe this type of instrument to be the best choice for accurate quantitative ground-based measurements of the $5200 \AA$ nightglow.

R. G. Burnside* J. W. Meriwether, Jr. $\dagger$ M. R. Torr $\ddagger^{*}$

*National Institute for Telecommunications Research, C.S.I.R., P.O. Box 3718, Johannesburg, South Africa. $\dagger$ Arecibo Observatory, P.O. Box 995, Arecibo, PR 00612, U.S.A.

¥ Space Physics Research Laboratory, University of Michigan, Ann Arbor, MI 48105, U.S.A.

$\$$ National Astronomy and Ionosphere Center, Comell University, Ithaca, NY 14853, U.S.A.

Acknowledgements-We thank Dr. J. C. G. Walker for his encouragement and for reading the manuscript, and Dr. Fred Herrero and Mr. Raul Garcia for their assistance. The loan of the one metre Ebert-Fastie spectrophotometer to Arecibo Observatory by the Kitt Peak National Observatory is gratefully acknowledged. NAIC is operated by Cornell University under contract with the National Science Foundation.

\section{REFERENCES}

Broadfoot, A. L. and Kendall, K. R. (1968). The airglow

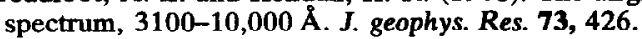

Curcio, J. A., Drummeter, L. F. and Knestrick, G. L., (1964). Atlas of the absorption spectrum of the lower atmosphere from $5400 \AA$ to $8520 \AA$. Appl. Opt. 3, 1401.

Garstang, R. H. (1956). The Airglow and the Aurora. (Eds. E. B. Armstrong and A. Dalgarno), p. 324. Pergamon Press, Oxford.

Hernandez, G. (1974). Contamination of the OI $\left({ }^{3} P_{2}-\right.$ ${ }^{1} D_{2}$ ) emission line by the (9-3) band of $\mathrm{OH} X^{2} \Pi$ in high-resolution measurements of the night sky. $J$. geophys. Res. 79, 1119.

Hernandez, G. and Turtle, J. P. (1969). The NI $\left({ }^{4} S_{3 / 2}^{0}-\right.$ ${ }^{2} D_{5 / 2,3 / 2}^{0}$ ) transitions in the upper atmosphere at night. Planet. Space Sci. 17, 675. 